\title{
SINDACATO E PANDEMIA. \\ INTERVISTA A CHRISTIAN FERRARI SEGRETARIO CGIL VENETO
}

\author{
di Anna Carreri*e Nicoletta Masiero**
}

Mercoledì 16 settembre 2020, con un confronto via Skype, Anna Carreri e Nicoletta Masiero hanno intervistato Christian Ferrari, segretario Cgil Veneto (nell'intervista: ChF). All'intervista, che rientra nell'ambito della ricerca Lavoro: organizzazione e vita lavorativa. Quale ruolo del sindacato e della contrattazione collettiva, ha partecipato anche Giorgio Gosetti che coordina il progetto condotto da Università di Verona e Ires Veneto.

D - Per la ricerca Lavoro: organizzazione e vita lavorativa che stiamo conducendo con i sindacalisti di Fisac, Fillea, Filcams, nei settori bancario, edile e della grande distribuzione organizzata, abbiamo pensato che fosse necessario integrare il materiale già raccolto fino a gennaio 2020 con un'esplorazione post lock-down. La portata di quanto avvenuto in questi mesi ci ha indotto a rivedere il progetto di ricerca per capire se il confinamento e le misure prese per contrastare l'emergenza Covid-19 abbiano inciso e in che modo su alcune questioni emerse che indicavano il bisogno che il sindacato ripensi ai mezzi, ai contenuti, alle modalità tipiche del fare attività sindacale. Capire quali tendenze si sono accentuate, oppure no e in che senso. Pensi si possa parlare di una frattura, di un prima e di un dopo il lock-down?

ChF - La frattura c'è stata sicuramente, e in tutte le dimensioni della vita sociale, civile, pubblica e anche della vita di ciascuno di noi. Lo percepiamo tutti quanti: oltre che terribili, questi sono stati mesi di traumatico cambiamento, una specie di spartiacque storico che ha cambiato in profondità gli stili di vita e quindi anche le modalità dell'azione sindacale. Per dirla plasticamente: il fatto che il sindacato con difficoltà riesca ad andare, per esempio, a tenere delle assemblee, oppure, un altro esempio, il fatto che venerdì prossimo (18 settembre) la prima manifestazione sindacale regionale unitaria di Cgil, Cisl e Uil in costanza di Covid si svolgerà con modalità del tutto diverse rispetto a quelle a cui eravamo abituati, fotografa una realtà che è già sotto gli

* Università degli Studi di Trento e Università di Hasselt, Belgio.

** Ires Veneto, Venezia.

ECONOMIA E SOCIETÀ REGIONALE - ISSN 1827-2479 - XXXVIII(2) 2020 - COVID-19 E LAVORO: SGUARDI INTERDISCIPLINARI DOI: $10.3280 / \mathrm{ES} 2020-002015$ 
occhi di tutti. Noi finora siamo sempre stati degli "animatori di assembramenti", e molto contenti di esserlo; adesso dobbiamo gestire distanziamenti anche nelle mobilitazioni di piazza, che sono una delle forme con cui il sindacato ha storicamente portato avanti la propria iniziativa politica. Quindi è chiaro che siamo di fronte, in termini più complessivi, ma anche per quanto riguarda noi come organizzazioni sindacali, a un momento che impone una profonda riflessione. Penso, anzi, che al sindacato si richieda un'ulteriore accelerazione dei processi e delle tendenze di cambiamento che erano già in atto. Quindi, la crisi Covid come catalizzatore, acceleratore di tendenze, di processi e di contraddizioni già presenti da tempo. Vale in generale per il mondo, per la dimensione sociale e politica, e vale anche per il sindacato. I ritardi del sindacato sono arrivati al pettine. Tutto ciò, peraltro, rappresenta un carico da novanta che si somma alle tre grandi trasformazioni che già ci stanno attraversando: quella tecnologica, quella ambientale, quella demografica. Sono le tre grandi sfide degli anni Venti, rispetto alle quali il sindacato evidentemente deve prepararsi per affrontarle nei termini giusti, cercando di incidere sul processo di cambiamento.

D - Per passare allo specifico di quanto abbiamo rilevato, partiamo da una prima questione che ci è stata segnalata e che possiamo indicare come la frattura generazionale, che riporta in parte anche alla trasformazione demografica di cui hai appena detto. È risultata particolarmente avvertita la difficoltà, e al contempo la necessità, del sindacato di avvicinarsi alle fasce più giovani, che hanno un approccio al lavoro molto diverso rispetto ai lavoratori più maturi e, soprattutto, una minor capacitàlpropensione nel rivendicare diritti collettivi. In questo senso, come ha impattato l'esperienza del lock-down e della pandemia? È possibile ricavarne indicazioni di percorso?

$\mathbf{C h F}$ - Dobbiamo pensare e realizzare un sindacato più inclusivo. La frantumazione del mondo del lavoro è un processo iniziato negli anni Novanta e certo... noi ce n'eravamo già accorti, ma l'emergenza Covid, anche in questo caso, è stata un detonatore. La precarietà, il lavoro povero e individualizzato fanno sì che tanti giovani incrocino, con maggiore difficoltà che in passato, la dimensione della rappresentanza collettiva. Parlo di condizioni materiali per sottolineare che sono quelle che determinano certi comportamenti di tipo individualistico. Non è che i giovani non capiscano, non si interessino di sindacato perché pensano di essere imprenditori di sé stessi. Per il sindacato questa è una doppia sfida, sul piano contrattuale e su quello politico-legislativo. $\mathrm{Su}$ quest'ultimo abbiamo agito anche in questi anni, penso alla Carta dei diritti fondamentali del lavoro depositata in Parlamento, per cercare di ricomporre e riunificare il lavoro attraverso un'indispensabile inversione di tendenza rispetto a indirizzi legislativi che via a via hanno frantumato il mondo del lavoro 
negli ultimi trent'anni. Ma è certamente una sfida che parla anche a noi, alla nostra azione contrattuale e di tutela collettiva e individuale.

D - A proposito di questo, la difficolta del sindacato di fronte alla frattura generazionale si collega proprio a quell'altra difficoltà che ci è stata indicata, già in assenza di Covid-19, come problematica ossia la crescente eterogeneità della forza lavoro in ogni fascia d'età, perché sono sempre più diversificate le situazioni contrattuali e lavorative. Si è accentuata, con la pandemia, la necessità, già prima avvertita, che il sindacato si apra a nuovi obiettivi, andando oltre la tutela del lavoro subordinato standard, per cosi dire, rappresentando anche altre forme contrattuali e ponendo al centro anche temi di frontiera rispetto al lavoro come il tema dell'ambiente, della salute ...?

$\mathbf{C h F}$ - Guardate cos'ha voluto dire la crisi Covid per il lavoro precario e autonomo. Io sono tra quelli che pensa che questa tragedia sia anche, e non è retorica, una straordinaria occasione di cambiamento da affrontare con l'ottimismo della volontà. Per la prima volta, la politica è stata costretta a misurarsi con una larga fascia di mondo del lavoro che sostanzialmente era abbandonata a sé stessa. Per la prima sono stati attivati ammortizzatori sociali universali lo dico in senso a-tecnico - che hanno coperto anche il mondo del lavoro autonomo, le partite Iva. Lì ci sono un vuoto di rappresentanza e un nostro ritardo che vanno colmati. La prima occasione è quella, che stiamo sollecitando, di una riforma strutturale, e in senso inclusivo e universale, del sistema degli ammortizzatori sociali, che finalmente dia piena copertura a tutto il mondo del lavoro. E questa non è solo una rivendicazione verso la politica, o un obiettivo strategico della nostra azione contrattuale, è anche l'occasione per far incontrare il sindacato a questo vasto mondo che, oggettivamente, si è sempre sentito altro. È lo stesso discorso che vale per i giovani: non è una questione solo di proselitismo o di ingegneria organizzativa, dobbiamo fare e sostenere politiche che parlino e che incidano su quelle condizioni, sulle condizioni del lavoro precario e del lavoro autonomo, ovviamente parlo del lavoro autonomo "povero", delle partite Iva economicamente non indipendenti. Questo è sicuramente un tema che deve tornare al centro. Così come l'allargamento del perimetro e del target della nostra contrattazione collettiva. Faccio l'esempio dei riders. Tra l'altro, lavoratori che nel pieno del lock-down, nel momento più drammatico, sono stati protagonisti straordinari della tenuta sociale, economica e civile del Paese. Quando i più se ne stavano a casa, il lavoro più faticoso, più povero, più precario ha dato un contributo decisivo a tenere insieme l'Italia. Il lavoro dei riders è un esempio, ma rappresenta anche un fronte concreto di azione contrattuale che stiamo portando avanti. La questione è riconoscere a questo lavoro la dignità e i diritti di un lavoro vero e proprio, con una disciplina collettiva, contrattuale e così via. Il sindacato si 
deve occupare anche di queste condizioni sociali, non può rimanere chiuso esclusivamente nell' ambito del lavoro standard, che pur rappresenta il suo naturale $-\mathrm{e}$ fondamentale - insediamento sociale. Bisogna però capire che oggi quello del lavoro precario e disperso è il bisogno sociale più acuto. In questo senso, permettetemi un parallelo con la bilateralità. Nel settore frammentato dell'artigianato del Veneto, attraverso questo strumento, abbiamo incluso un insieme disperso di lavoratori, che quasi sempre non aveva l'alternativa di una dimensione aziendale di contrattazione, in un ambito di tutele e di diritti prima sconosciuti. Con altri strumenti, dobbiamo fare lo stesso per i lavoratori precari e autonomi.

D - Queste due prime questioni - frattura generazionale ed eterogeneità della forza lavoro - spingono da tempo il lavoratore a una domanda di servizi e di tutela personale, che richiama un dilemma divenuto annoso sulla missione del sindacato all'interno del sindacato stesso... E, unitamente a questo, la tendenza del lavoratore a personalizzare il rapporto con il delegato sindacale, il quale in un certo senso si trova ad essere iper-responsabilizzato. In particolare, durante il lock-down, a noi risulta che il sindacalista di territorio ha avuto un sovraccarico di richieste di lavoro quasi ingestibili (umanamente incontenibili) e moltiplicate enne volte, in quanto ha dovuto ripetere le stesse cose con telefonate e videochiamate individuali a ogni richiesta anziché dirle una volta per tutte a tutti i lavoratori riuniti in un'assemblea. Se nella cassetta degli attrezzi oggi ci sono WhatsApp, Skype, Facebook, Instagram ..., si pone il problema anche per far fronte a un'azione sindacale sempre più personalizzata e tecnologica. A che punto siamo su questo? Ci sono delle strategie in cantiere, se non già attivate, per ripensare il lavoro stesso del sindacalista?

ChF - Durante il lock-down, nelle tragiche condizioni date, è venuta fuori tutta la passione, la generosità, lo spirito di militanza che connota i dirigenti, gli operatori, i militanti, i delegati e i pensionati della Cgil. È stato fatto un lavoro straordinario che ha garantito la tenuta e la continuità di azione - nelle forme e nei modi possibili - di tutte le nostre strutture. Se nell'ora più buia per il Paese e per le nostre comunità la Cgil è stata un punto di riferimento per le persone, per le istituzioni, per la politica, lo si deve innanzitutto a questo lavoro eccezionale che quotidianamente le nostre compagne e i nostri compagni hanno portato avanti. Una cosa che personalmente non dimenticherò: nel momento più drammatico ho sentito, come non mai, l'orgoglio di appartenere a questa organizzazione. Ed è vero che in questo sforzo inedito dal punto di vista organizzativo, politico e umano, ci sono stati momenti in cui qualcuno è stato sull'orlo del burnout. I sindacalisti sono donne e uomini in carne e ossa, come lo sono i medici, gli infermieri, le commesse e tutti quei lavoratori principalmente donne - che hanno assicurato le attività e i servizi essenziali 
in quei mesi. È stata una cosa travolgente. Poi magari non si conosce, non si vede dall'esterno ma la Cgil è stata un punto decisivo di tenuta del Paese. Le nostre sedi - pur applicando tutte le misure restrittive assunte dal Governo sono sempre state aperte. L'azione di tutela e rappresentanza non ha mai visto un momento di tregua. I nostri servizi e la tutela individuale hanno continuato a dare risposte alle persone anche attraverso gli strumenti tecnologici, che per fortuna ora ci sono! Una pandemia come questa, soltanto dieci o quindici anni fa, negli anni 2000, avrebbe certamente avuto un impatto molto più devastante. Penso in particolar modo ai nostri Caaf e al Patronato Inca, da tempo il nostro laboratorio di innovazioni tecnologico-organizzative. E nel momento del bisogno questo patrimonio e questa esperienza sono stati fondamentali. In generale stiamo portando avanti molti progetti di innovazione sul piano degli strumenti informatici, dei portali, delle banche dati, della digitalizzazione, per migliorare e rafforzare il più possibile il rapporto con le persone anche in questo modo. L'obiettivo è una sempre maggiore integrazione tra la tutela collettiva e quella individuale, tra le categorie e il sistema servizi. Abbiamo superato definitivamente la falsa contrapposizione tra queste due dimensioni di tutela, ponendo fine alla lunga e stucchevole discussione circa una presunta (e inesistente) dicotomia fra il sindacato dei servizi e il sindacato dei diritti. Non esiste, è una falsa antitesi. Il Sindacato è uno solo: quello della contrattazione, quello dei diritti e dei bisogni di lavoratori e pensionati, quello che, tutelando e migliorando le loro condizioni, ha un progetto più generale che punta a trasformare l'intera società. E per fare ciò utilizza al meglio tutti gli strumenti e le leve di cui dispone. Tutela collettiva e individuale sono entrambe dimensioni indispensabili che si completano e si rafforzano a vicenda. Pensiamo in un mondo del lavoro oggi parcellizzato e individualizzato all'importanza che rivestono i nostri servizi per poter intercettare un'ampia fascia di bisogni e di soggetti che altrimenti non incrocerebbero mai il sindacato. Poi certo, sta a noi realizzare una "presa in carico" anche politica di queste persone, e questo rappresenta sicuramente un punto critico su cui dobbiamo sforzarci e lavorare di più. Ma per tornare alla domanda: non c'è dubbio che, anche per il sindacato, Covid-19 ha fatto emergere l'importanza strategica della tecnologia. Durante il lock-down, per le casse integrazioni è stato fatto un miracolo. Tra l'altro ricordo che nel Veneto, a differenza del resto d'Italia, siamo riusciti a imporre l'obbligo di informativa e di accordo (per quanto con procedura semplificata) anche per le richieste di cassa in deroga sotto i cinque dipendenti. A un certo punto le nostre strutture sono state letteralmente investite da una valanga di Pec. Caaf e Inca non si sono mai fermati grazie alla tecnologia. Dalle prestazioni "ordinarie" a quelle legate ai nuovi provvedimenti di tutela varati dal governo. E abbiamo dato risposte, preso in carico moltissime persone, garantito l'esercizio di diritti. L'abbiamo fatto sia con il rapporto diretto che con 
quello telematico. La potenzialità di questi strumenti è evidente anche a chi magari prima dell'emergenza Covid era scettico. Dobbiamo integrare sempre più queste dimensioni che rappresentano le facce di una stessa medaglia, formando sindacalisti a tutto tondo, che siano in grado di dare risposte a $360^{\circ}$ ai bisogni e alla domanda di tutela delle persone.

D - Restiamo in tema di tecnologico e digitale anche passando a un'altra questione critica: la "comunicazione" del sindacato. Utilizzando il termine "comunicazione" come capacità di veicolare contenuti, obiettivi, di diffonderli, di raggiungere iscritti e non iscritti, non ovviamente nel senso deteriore di trasmissione massmediatica e acritica di messaggi privi di determinazione. Rispetto a questo, prima che scoppiasse la pandemia, abbiamo individuato riserve e perplessità sia sulla capacità di comunicare del sindacato, che si inserisce in un quadro generale di disaffezione per la politica e per $i$ corpi intermedi, sia su una certa arretratezza dei mezzi di comunicazione utilizzati. Ecco questo duplice fronte, come si configura dopo l'emergenza Covid?

ChF - Oltre a quanto detto, l'esperienza Covid-19 ci indirizza sicuramente verso un sindacato più digitale. Non è uno slogan, è un'urgente necessità. Anche noi, sull'onda dell'emergenza, abbiamo vissuto un'accelerazione nell'utilizzo degli strumenti digitali che, vi assicuro, non è stata seconda ad altri ambienti e contesti. Siamo riusciti, attraverso la tecnologia, a garantire una continuità della discussione, del confronto e anche dell'iniziativa dei gruppi dirigenti, e in qualche modo anche del rapporto con i lavoratori. E penso che questo sia un punto da cui non si torna indietro. Va colto e sviluppato come grande occasione di innovazione dell'azione sindacale. Non certo per superare e sostituire le forme, i luoghi e le modalità più tradizionali della rappresentanza: la presenza fisica e il contatto diretto con le persone. Non appena ne avremo le condizioni, speriamo quanto prima, non vediamo l'ora di ritornare con ancor maggiore intensità nei luoghi di lavoro, che pure non abbiamo mai smesso di frequentare seppur limitati dalle misure di tutela della salute. Quindi, la digitalizzazione del sindacato non per sostituire, ma per rafforzare e completare le modalità tradizionali che non andranno mai abbandonate. Perché sarebbe un errore illudersi che tutto possa semplicemente essere traslato in un'esclusiva dimensione social e digitale. Anche queste non sono dimensioni contrapposte ma complementari. E allora: avanti tutta con le video-call, con i servizi digitali, con i big data che rappresentano una grande opportunità nella nuova era. Per il sindacato, pensate agli iscritti, agli utenti, a tutti i soggetti con cui entra in relazione e alle potenzialità ovviamente non commerciali, ma politiche e sindacali che possono svilupparsi attraverso una gestione più efficace, più professionale e anche più avanzata e innovativa di quel tesoro che sono le nostre banche dati. $\mathrm{E}$ in termini di innovazione organizzativa delle 
nostre strutture, compresa anche la comunicazione, che non sottovaluto, soprattutto se vuoi parlare a una certa fascia della società e del mondo del lavoro, in particolar modo quella più frammentata, quella più giovane, anche i social network diventano uno strumento di azione politica e sindacale. Abbiamo strategicamente, negli ultimi anni, investito molto su quest'ultimo ambito come strumento di diffusione delle nostre posizioni, delle nostre idee, delle informazioni, dei servizi che forniamo e siamo arrivati, proprio la settimana scorsa, a superare i 10.000 like sulla pagina Facebook della Cgil Veneto, con un potenziale di diffusione istantanea che, anche solo qualche anno fa, era impensabile poter fare in maniera così facile ed economica. Ma non ci siamo limitati a Facebook, siamo su Twitter, su Instagram, utilizziamo Telegram per informare e aggiornare il nostro gruppo dirigente e l'apparato. Nei giorni più difficili dell'emergenza sanitaria, sui social le nostre prese di posizione hanno raggiunto centinaia di migliaia di persone, molte di più di quanti sono i lettori dei quotidiani locali, su cui comunque sono comparsi i nostri comunicati e le nostre dichiarazioni. Tutto ciò non è in contrapposizione al fatto che nella bacheca delle nostre fabbriche continuino ad esserci i volantini, i comunicati e tutto il resto. Sul piano della comunicazione vale ancora di più ciò che dicevo prima: o ti digitalizzi o non tocchi palla.

Guai però a contrapporre la digitalizzazione al radicamento nei territori. Noi, a differenza dei partiti, non abbiamo fatto l'errore esiziale di cedere alle sirene della politica liquida. Mi riferisco evidentemente agli ultimi vent'anni, nel corso dei quali la politica mediatica ha via via sostituito la presenza nei territori (le famose sezioni) e il contatto diretto con le persone. Noi abbiamo scelto la strada opposta e credo che abbiamo fatto non bene, ma benissimo perché, se siamo sopravvissuti come soggetti collettivi di rappresentanza, anzi in Veneto siamo addirittura cresciuti, mentre le forze politiche sono diventate quasi esclusivamente dei comitati elettorali, lo dobbiamo alla decisione strategica di aumentare e non diminuire la nostra presenza nei territori. Quindi la presenza diffusa, il radicamento capillare nei posti di lavoro e nel territorio, continueranno ad essere la strada giusta da percorrere anche in futuro. Lavoratori e pensionati hanno bisogno di un punto di riferimento fisico, di persone in carne e ossa con cui parlare, e poi di trovare anche sul web la posizione del loro sindacato. Altrimenti la sola presenza digitale rischia di essere un guscio vuoto, qualcosa di effimero. Quindi, per dirla con uno slogan, dobbiamo essere, al contempo, un sindacato digitale e di strada, solo così potremo coprire tutte le dimensioni del lavoro e della vita sociale.

D - Una maggior presenza quindi sui luoghi di lavoro per parlare ai lavoratori e alle lavoratrici che non sono a conoscenza di cosa sia il contratto collettivo, ad esempio, né cosa fa e cosa sia il sindacato, come ci è stato detto, 
questa è un'esigenza. Ma ci è stato raccontato anche che il problema della difficoltà a farsi conoscere è anche fuori dal posto di lavoro...

ChF - Infatti: non solo azienda, ma territorio. Covid ci dice anche questo. La dimensione della fabbrica e la dimensione fuori dai cancelli, del territorio, devono sempre più intrecciarsi e coordinarsi. Sono valutazioni, inviti che rivolgo a noi stessi. Perché ciò accada dobbiamo uscire da una dimensione di contrattazione esclusivamente chiusa nei perimetri del luogo di lavoro e del contesto lavorativo. Il potenziamento del welfare dobbiamo ottenerlo nel territorio, dalle istituzioni, molto più del welfare aziendale! Perché per noi lo stato sociale deve evitare una deriva che è opposta alla solidarietà ed essere un valore universale di cui ci sarà sempre più bisogno. La conciliazione tra tempi di vita e di lavoro dipende anche dai tempi delle città, dai servizi che il lavoratore trova fuori dalla fabbrica. Questo è un grande tema su cui dovremo concentrarci per essere pronti ad affrontarlo con maggior attenzione.

D - A proposito di organizzazione del lavoro, durante il lock-down, la sospensione del lavoro in molte realtà produttive e soprattutto il ricorso massiccio a modalità prima quasi inedite o raramente applicate come il lavoro da casa portano in primo piano la necessità di attrezzarsi per affrontare nuovi modelli organizzativi delle imprese. Come impatta questo sui contenuti e sugli obiettivi dell'azione sindacale?

$\mathbf{C h F}$ - Una delle cose che ci dice questa crisi è che per il sindacato è fondamentale ritornare a parlare, a incidere, a contrattare l'organizzazione del lavoro e della produzione. Questo è per noi, in parte, un buco nero. Non vorrei peccare di eccessiva autocritica, ma l'organizzazione del lavoro è da trent'anni che l'abbiamo abbandonata. Per carità, dal 2008 in poi, quindi sull'onda di una stagione difensiva, le priorità sono state drammaticamente altre. Oggi, la crisi Covid ripropone all'ennesima potenza un tema che, per me, che sono un "trentiniano", è il cuore dei rapporti di produzione capitalistica, come si sarebbe detto un tempo. Pensate al tema della salute e sicurezza durante l'emergenza sanitaria. Drammaticamente è piombato al centro dell'agenda delle relazioni sindacali e anche della politica. E la battaglia che abbiamo fatto è stata affermare il principio di civiltà secondo cui la salute viene prima del profitto, mentre una parte rilevante della società italiana e delle nostre controparti, magari senza esplicitarlo, questo principio hanno tentato di negarlo. Basti pensare a Bergamo e Brescia, che sotto questo punto di vista - con le mancate chiusure causate dal mix micidiale di una politica miope e una Confindustria irresponsabile, rimarranno una pagina nera nella storia italiana. È questo principio che ci ha permesso, sulla base di esigenze e di interessi primari come la salute delle persone, di ritornare a mettere il naso nell'organizzazione del lavoro e della produzione all'interno delle imprese. I 
comitati aziendali che abbiamo conquistato nei protocolli sottoscritti a livello nazionale, a livello regionale, e poi declinati in ciascun luogo di lavoro sono stati fondamentali per proteggere la nostra gente, aprendoci peraltro un varco per riconquistarci un ruolo che avevamo perso da tempo. Il tema che si pone per il sindacato in questo nuovo scenario è andare avanti e porre al centro delle rivendicazioni la partecipazione dei lavoratori - quella prevista dall'articolo 46 della Costituzione - perché non c'è nulla di nuovo da inventarsi, è come suol dirsi un "ritorno al futuro". Più protagonismo sia nelle strategie industriali della singola azienda, sia nelle politiche industriali pubbliche, che noi consideriamo un altro elemento assolutamente indispensabile della nuova stagione di cambiamento necessaria per superare la crisi e costruire un mondo del lavoro migliore per i lavoratori e per il nostro Paese. In questo ambito rientra anche lo smart-working. Non c'è dubbio che, proprio in considerazione delle sue luci e delle sue ombre, questa modalità di lavoro deve essere ri-contrattualizzata dal sindacato quanto prima, non appena sarà superata la legislazione dell'emergenza. Se allarghiamo ancora di più il campo, arriviamo alla rivoluzione tecnologica, che non può essere un processo spontaneo (che poi spontaneo non è, visto che fin qui è stato nelle mani di pochissimi), ma va declinato socialmente, va gestito e governato e a monte per non subirlo a valle. E ci vuole un protagonismo collettivo dei lavoratori. Questo valeva prima dell'emergenza Covid e vale ancor di più adesso e per il dopo.

Noi stiamo andando verso una tragedia occupazionale di dimensioni mai viste prima. Non appena salterà il divieto di licenziamento e terminerà la fase straordinaria degli ammortizzatori sociali, la crisi occupazionale, potenzialmente dirompente, si combinerà con le conseguenze della rivoluzione tecnologica che, come noto, porta - anche se non in maniera omogenea - a un aumento della produttività enorme. Rispetto a questo combinato disposto, che porterà a perdita ingente di lavoro, è evidente che noi dobbiamo, soprattutto, puntare sulla riduzione dell'orario di lavoro (non di salario) per ridistribuire sia il lavoro che il valore aggiunto prodotto. Non vedo altro rimedio, d'altronde questa è una costante di qualsiasi passaggio di fase negli ultimi due, tre secoli per il mondo del lavoro.

D - Si parla di una spaccatura tra imprese piccole e grandi, ad esempio nell'agricoltura. Soprattutto le imprese grandi-ci dicono - hanno chiesto un contributo al sindacato, di stare dentro e di collaborare alla gestione di problemi come l'ansia. Forse l'emergenza Covid ha fatto capire che su alcune cose concrete e pratiche, che poi possono diventare collettive nei luoghi di lavoro, il sindacalista ha avuto un ruolo molto importante.

ChF - Confermo, è stato proprio così. Poi, passata l'emergenza, diciamo che c'è la tentazione di far tornare ciascuno alle proprie postazioni. È un errore, 
perché la partecipazione dei lavoratori - che non è quella finanziaria, e neanche la presenza nei Cda, per carità, può avere anche quel tipo di modalità, ma non è quello il punto - vuol dire soprattutto co-determinare i piani, i progetti, gli sviluppi, le scelte di strategia industriale. È un tema, a maggior ragione in un ambiente tecnologicamente più innovativo e avanzato, che sarà ineludibile. Oggi il coinvolgimento si limita al massimo alla singola professionalità e invece deve trovare una dimensione collettiva, con la partecipazione di tutti i lavoratori interessati. Se succedesse, sarebbe un bene per tutti. Ma fin qui è stato difficilissimo anche solo discuterne con le nostre controparti. Noi siamo disegnati come quelli "antichi", conflittuali, che si occupano solo del binomio orario/salario - che comunque sarà sempre cruciale in un sistema capitalistico - ma quando proviamo ad andare oltre i primi a irrigidirsi sono gli imprenditori. Proprio coloro che partecipano un giorno sì e l'altro pure ai convegni sull'innovazione, sulla partecipazione, su un ambiente lavorativo più friendly... Quando proponiamo di realizzarlo veramente, sono loro che non ci stanno. Su questo tema sto discutendo molto con i miei omologhi di Cisl e Uil, in particolare con la Cisl, perché vorremmo cogliere quest'occasione per cercare anche di organizzare un'azione concreta a livello Veneto.

D - Da quanto dici va ripensato il sindacato, il modo di fare sindacato, mettendo insieme tutti i fattori di cui abbiamo parlato (Covid, digitale...). Una straordinaria occasione per farlo e se non lo si fa ora si rischia di restare invischiati in categorie interpretative obsolete, modi di pensare inefficaci. $\grave{E}$ un po' come se fossero saltate quelle opposizioni che strutturavano anche delle false certezze: tutele, servizi da una parte e sindacato da un'altra, oppure la falsa contrapposizione lavoro e salute, uno dei tormentoni con quello dell'opposizione fra ambiente e lavoro. Sono opposizioni che vanno riconsiderate alla luce di quanto è stato accelerato dall'emergenza Covid... da intendersi appunto come acceleratore di processi precedenti. Forse si apre una grande stagione di formazione per il sindacato, sui modelli organizzativi....

ChF - Sono assolutamente d'accordo. Tra l'altro, proprio per uscire da una dimensione ideologica e non limitarci agli slogan, i contesti industriali più forti e più innovativi, sono anche i contesti in cui la contrattazione è più sviluppata. Qualità della produzione, qualità della contrattazione, qualità del lavoro sono fattori profondamente intrecciati tra loro. E la vera sfida che sta di fronte al sistema produttivo italiano è la stessa da ormai trent' anni, ed è il bivio tra la via alta e la via bassa alla competitività e allo sviluppo. È una sfida che fin qui abbiamo perso, non rendendoci conto che scegliere la via alta, quella che punta su innovazione, ricerca e qualità del lavoro e del prodotto, è davvero un gioco win-win per tutte le parti coinvolte. Le realtà dell'industria avanzata 
tedesca, che tra l'altro trova molto attrattivo l'ambiente italiano, il lavoro italiano, a dispetto della nostra tendenza all'autodenigrazione, lo dimostra. E penso che oggi, tra le tante cose, abbiamo l'occasione, gli strumenti e le possibilità proprio per ripensare il nostro modello e scegliere una strada diversa rispetto a quella percorsa negli ultimi decenni. Penso che, da questo punto di vista, richiamando ancora Bruno Trentin, anche il sindacato debba dare un suo contributo per spingere in questa direzione. Più che attardarsi su battaglie di retroguardia, dobbiamo accettare di essere attraversati da questa grande trasformazione, soprattutto tecnologica. Abbiamo delle condizioni irripetibili sul piano delle risorse, cosa che non succedeva da moltissimo tempo. Sul piano delle condizioni politiche, della cultura politica, si stanno smontando totem e dogmi ideologici consolidati. L'illusione neoliberista dell'autoregolamentazione del mercato, il meno Stato più mercato, le mani libere da lasciare alle imprese e così via. Adesso si è riproposta brutalmente la centralità dell'intervento pubblico in termini di welfare universale e anche in termini di governo dell'economia. Anche in questo caso mi vien da definirlo "un ritorno al futuro": vuol dire reinterpretare, alla luce dei tempi che viviamo, quel modello di economia mista che rappresenta il cuore della nostra Costituzione. Non sto pensando di tornare alle partecipazioni statali degli anni Settanta, sto pensando a Mariana Mazzucato e molti altri economisti, e oggi ci sono le risorse e anche le condizioni politiche per una svolta in quella direzione. E mi preoccupa molto l'atteggiamento difensivo e di retroguardia di alcune nostre controparti. Della nuova Confindustria di Bonomi mi colpisce che la proposta per uscire dalla crisi e costruire una nuova Italia è la stessa vecchia ricetta che ha segnato il lungo declino industriale italiano, quella parabola discendente iniziata negli anni Novanta con la dissennata stagione delle privatizzazioni e della rinuncia deliberata alla Politica industriale. La verità è che anche prima dell'emergenza Covid non stavamo per niente bene. E allora, altro che tornare al "meno Stato più mercato", c'è piuttosto da riscoprire la forte complementarità tra l'intervento pubblico e l'iniziativa privata, tra la grande industria a partecipazione pubblica e il sistema delle imprese privato. Quella integrazione, quella complementarità sono state la cifra del modello di economia mista che ci ha fatto diventare - nel giro di vent'anni - la quarta potenza industriale del mondo, ricostruendo dalle macerie della Seconda guerra mondiale.

D - Questo ripensamento del sindacato richiede forse anche un ripensamento del rapporto fra confederale e categorie (comparti, corporazioni), spesso lontane dalla concretezza delle dinamiche che osserviamo ogni giorno, nel senso che richiede di rivedere le suddivisioni della rappresentanza e dell'organizzazione merceologica? 
$\mathbf{C h F}$ - Abbiamo bisogno di un sindacato sempre più confederale e orizzontale. Questa è una nostra caratteristica storica, potremmo definirla la matrice della Cgil e del sindacalismo italiano. La stessa crisi che viviamo ci chiede di essere sempre più confederali. È necessaria una riforma profonda anche della Cgil, del suo modo d'essere, della sua forma, della sua struttura, che riflettono un mondo del lavoro e delle categorie merceologico-produttive degli anni Settanta. Fino ad ora, nonostante tutto, la bravura delle compagne e dei compagni, la nostra capacità di resilienza e flessibilità, ci hanno permesso di stare dentro a questo vestito stretto senza stracciarlo. Adesso però si pone l'urgenza di un cambiamento del modo d'essere della nostra organizzazione. Meno contratti nazionali, intendo dal punto di vista numerico, non meno contrattazione nazionale; passare da una logica di comparto a una logica di filiera produttiva, per governare in qualche modo la realtà e l'evoluzione della produzione. Se non cambiamo in questa direzione, il rischio è quello di riprodurre, di riflettere e amplificare proprio quella frantumazione del mondo del lavoro che, a parole, diciamo di volere affrontare e ricomporre.

E poi ci sarebbe da parlare anche del sindacato più partecipativo, che a mio giudizio vuol dire rafforzare e rilanciare il ruolo del delegato sindacale nel posto di lavoro. E qui c'è da fare anche un lavoro di formazione e culturale. Il sindacato deve essere di più agente culturale, anche nei confronti del proprio insediamento sociale. In questo modo si contribuisce a creare "capitale sociale", una delle cose che più manca a questo Paese. Una volta c'erano tante agenzie culturali, c'erano i partiti. I lavoratori arrivavano nel sindacato già strutturati, preparati, politicamente formati. Adesso non succede più, e penso soprattutto ai giovani. E non certo per colpa loro.

Chiudo davvero su un ultimo punto, una sfida di cambiamento che considero decisiva in prospettiva.

Abbiamo bisogno anche di un sindacato più internazionale, perché la dimensione dei problemi non solo non è regionale, ma non è neanche più nazionale, è quantomeno europea. E questo pone un'esigenza di innovazione a tutto il movimento sindacale, perché una proiezione continentale sarà sempre più indispensabile per incidere con un minimo di efficacia nei processi reali. E per conquistare una reale soggettività politico-sindacale internazionale serve lavorare per costruire una vera contrattazione collettiva europea, mentre oggi ciascuno chiuso dentro i propri confini - subiamo totalmente i fenomeni della finanziarizzazione, della svalutazione competitiva del lavoro, del dumping sociale tra i diversi Paesi. Un vero soggetto contrattuale - coordinato e unitario - a livello europeo darebbe anche un grande contributo politico e culturale a chi ha ruoli e responsabilità istituzionali nell'Unione e si batte per un'Europa diversa, più unità e più giusta. 